\title{
Annealing induced dissipation of residual trapped charge in Focused Ion Beam processed wide band gap materials for device applications.
}

\author{
Marion A. Stevens-Kalceff ${ }^{* * *}$ and Phillip Gowlett ${ }^{*}$ \\ *School of Physics, University of New South Wales, Sydney, 2052 NSW Australia. \\ ${ }^{* * *}$ Electron Microscope Unit, University of New South Wales, Sydney, 2052 NSW Australia.
}

Focused (keV) Ion Beam processing of wide band gap materials used in semiconductor device technology is an expanding and important area of research and technique development. For example, ion beam assisted deposition and sputtering processes enables the maskless modification of materials at the nanoscale for the fabrication or repair of specialized device structures.[1] When semiconductors and dielectrics are modified by a focused ion beam, localized charging may occur [2] and the assessment of the consequences of charging and associated physical processes must be assessed to enable optimal processing. In particular it is necessary to develop strategies to mitigate or minimize the ion beam induced localized modification of the specimen contact potential or specimen charging artifacts.

The effects of focused Gallium ion $\left(\mathrm{Ga}^{+}\right)$beam processing of Sapphire $\left(\mathrm{Al}_{2} \mathrm{O}_{3}\right)$, Silicon Carbide ( $\mathrm{SiC})$ and Quartz $\left(\mathrm{SiO}_{2}\right)$ crystals and in particular the resultant surface contact potentials were investigated. Each of these materials is an important component in various semiconductor and optoelectronic devices. The specimens were irradiated with a range of doses by systematically increasing processing time, such that a range of surface damage was observed from local swelling of a few nanometers of the surface due to beam induced amorphization, to sputtered holes hundreds of nanometers deep. Raman Spectroscopy showed peak broadening and attenuation as well as increased fluorescence from all processed materials, indicating defect generation consistent with ion beam induced amorphization of the crystalline structure. The irradiation induced changes in the Raman spectra increased as a function of dose. For example, a Raman emission associated with $\mathrm{Si}$-Si bonds was observed from the milled regions of Silicon Carbide, consistent with ion implantation induced carbon vacancy clusters with silicon chemistry.[3]

Kelvin Probe Microscopy (KPM) is a specialized Atomic Force Microscopy (AFM) technique in which long-range Coulomb forces between a conductive atomic force probe and a specimen surface enable the electrical potential at the specimen surface to be characterized.[4] AFM and KPM were employed to investigate the resultant topography and residual surface potentials resulting from the focused ion beam processing of these specimens. Ion beam induced residual surface potentials were observed to dissipate due to the passage of extended periods of time at controlled ambient conditions, and/ or due to in vacuo thermal treatments. Lower temperature $(<550 \mathrm{~K})$ thermal treatments not exceeding typical bakeout temperatures were investigated as a method to reduce charging induced effects, as it is usually undesirable to heat a device structure.

Significant dissipation of the residual potentials were separately observed resulting from either the passage of time or temperature treatments. The observed degree of dissipation was variable, ranging between negligible change and $70 \%$ attenuation depending on the material, FIB dosage, and thermal treatment temperature. Finite element modeling of the potential attenuation provides insight into the time and temperature induced changes in the in subsurface charge distribution [5]. It is proposed that 
processes associated with the attenuation of the residual potential include the thermally assisted electro-migration of electrons to regions of positive charge associated with implanted $\mathrm{Ga}^{+}$, and the relaxation of some shallow charge traps. However the relatively low temperatures of the thermal annealing treatments were insufficient to allow annealing of the ion irradiation induced lattice defects or restoration of the original Raman spectral profiles.

Focussed ion beam processing of wide band gap materials used as components in semiconductor and optoelectronic devices results in significant charging and/ or modification of the local contact surface potential difference. The passage of significant time and/ or lower temperature annealing treatments in vacuo reduce but are generally not sufficient to completely dissipate the ion beam induced effects.

\section{References}

[1]. International Technology Roadmap for Semiconductors (2004) http://public.itrs.net [2]. Stevens-Kalceff M.A., Rubanov S., Munroe P.R. "Kelvin Probe Microscopy of localised potentials induced in non-conductive materials by a Focused Ion Beam" in Microscopy of Semiconducting Materials 13. editors; Cullis A., Midgley P., Institute of Physics Conference Series V180. p 669-672. (2004).

[3]. Weber WH and Merlin R. "Raman Scattering in Materials Science" New York: Springer Verlag. (2000).

[4]. D. A Bonnell,. "Scanning Probe Microscopy and Spectroscopy: Theory, Techniques and Applications". Wiley-VCH. New York, (2001).

[5]. Programs HiPhi and Metamesh, Field Precision, Albuquerque, NM.

[6]. Support from the Australian Research Council is gratefully acknowledged. Corresponding author: Marion.Stevens-Kalceff@unsw.edu.au 